\title{
Modeling of Electron Temperature in Hypersonic Flows
}

\author{
Minkwan Kim* Ali Gülhan ${ }^{\dagger}$ \\ German Aerospace Center (DLR), Cologne, D51147, Germany \\ and Iain D. Boyd $\ddagger$ \\ University of Michigan, Ann Arbor, MI, 48109, USA
}

\begin{abstract}
Studies are described for an electron temperature model for hypersonic flow. The electron temperature is needed to be calculated separately, because it may have a significant effect on vibrational temperature and chemical reactions. Whenever flows are in a strong thermal nonequilibrium state, an electron energy equation should be considered. In the considered electron energy equation, the electron energy relaxations of each energy mode are accounted which include translational-electron, rotational-electron, and vibrationalelectron energy relaxation. In order to avoid the singularity of the Jacobian in the electron energy equation, we introduce a modified electron energy expression. The suggested electron temperature model is implemented into a hypersonic flow code for both explicit and implicit methods. In the present study, we numerically calculate the electron temperature with electron-vibrational relaxation for diatomic nitrogen. For the assessment of the electron temperature model, we simulate several cases which are a cylinder flow, a RAM-C case, the entry of the Automated Transfer Vehicle (ATV), and the Stardust reentry capsule.
\end{abstract}

\section{Nomenclature}

$e_{e} \quad$ Modified electron energy per unit mass, $e_{e}=\frac{\rho_{e}}{\rho} \varepsilon_{e}$

$\bar{R} \quad$ Universal gas constant, $8314.3[\mathrm{~J} / \mathrm{kg} \cdot$ mole $\cdot \mathrm{K}]$

$\dot{\omega}_{e} \quad$ Electron mass production rate by chemical reactions, $\left[\mathrm{kg} / \mathrm{m}^{3} \mathrm{sec}\right]$

$\lambda_{D} \quad$ Debye length, $[m]$

$\overrightarrow{J_{e}}$

$\overrightarrow{q_{e}}$

Electron diffusion flux

Electron heat flux

Flow velocity

Species density, $\left[\mathrm{kg} / \mathrm{m}^{-3}\right]$

$\rho_{s}$

$\sigma_{e s}$ Collision cross section for electron and $s$ species, $\left[\mathrm{m}^{2}\right]$

$\tau_{e s} \quad$ Electron-vibrational relaxation time, $[\mathrm{sec}]$

$\varepsilon_{0}$

$\vec{F}$

Vacuum permittivity, $8.854 \times 10^{-12}\left[C \cdot V^{-1} \cdot m^{-1}\right]$

Inviscid flux vector

$\vec{n} \quad$ Unit vector normal to computational cell face

$\vec{Q} \quad$ Vector of conserved variables

$b_{0} \quad$ Scattering parameter for $90^{\circ}, \frac{Z e^{2}}{12 \pi \varepsilon_{0} k T_{e}}\left[m^{2}\right]$

$e \quad$ Elementary charge, $1.6022 \times 10^{-19} \mathrm{C}$

$E_{e} \quad$ Electron energy, $E_{e}=\rho_{e}\left(C_{V, e} T_{e}+\frac{1}{2}\left(u^{2}+v^{2}+w^{2}\right)\right)$

$e_{e} \quad$ Electron energy per unit mass of electrons, $e_{e}=\left(C_{V, e} T_{e}+\frac{1}{2}\left(u^{2}+v^{2}+w^{2}\right)\right)$

$E_{i, f} \quad$ First ionization energy per unit mass, $[\mathrm{J} / \mathrm{kg}]$

* Research Scientist, Department of Supersonic and Hypersonic Technology, Institute of Aerodynamics and Flow Technology, min.kim@dlr.de, AIAA Member

$\dagger^{\dagger}$ Department Head, Department of Supersonic and Hypersonic Technology, Institute of Aerodynamics and Flow Technology, AIAA Member

${ }^{\ddagger}$ James E. Knott Professor, Department of Aerospace Engineering, AIAA Associate Fellow 


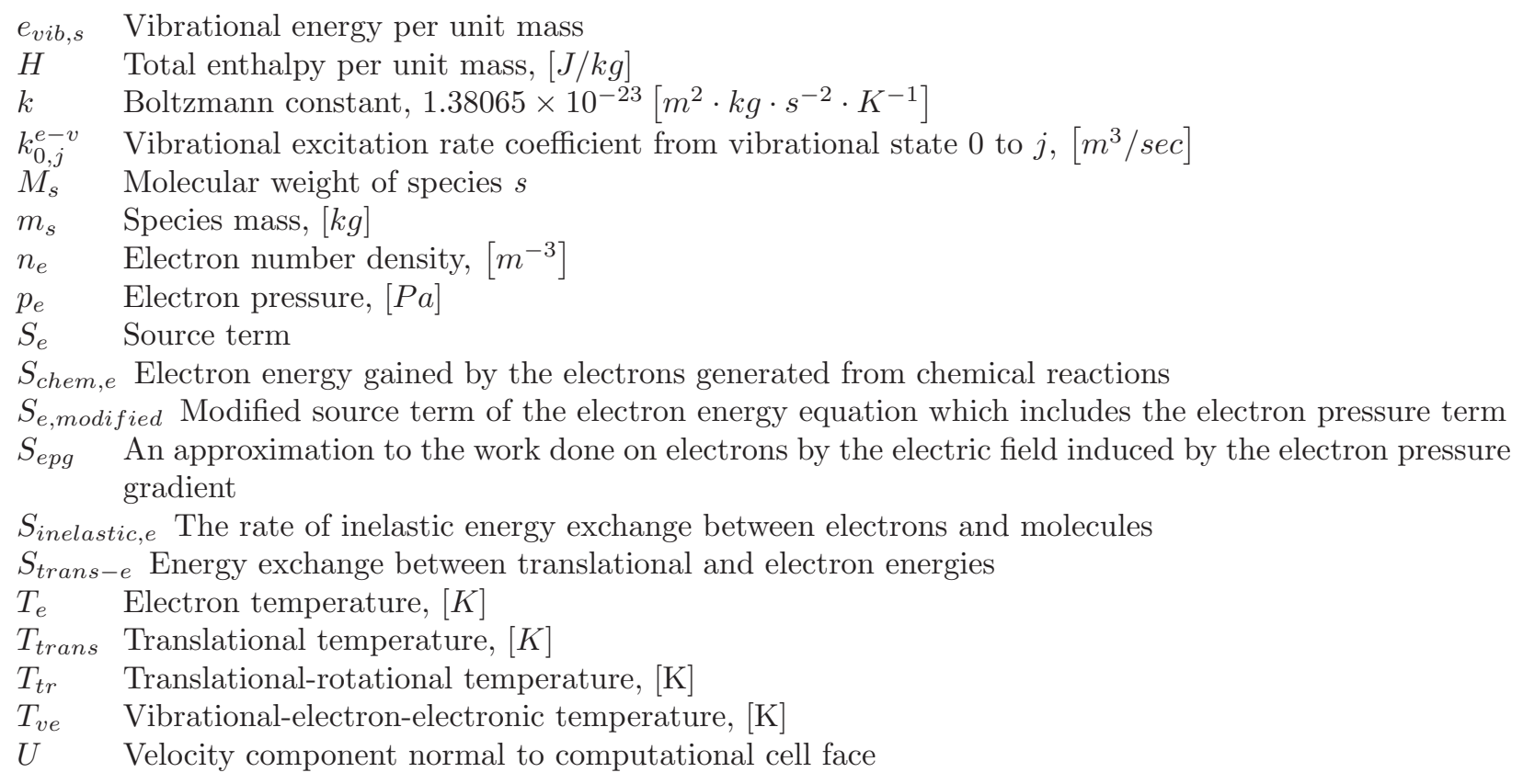

\section{Introduction}

A hypersonic flight vehicle has many applications for both military and civilian purposes including reentry vehicles such as the Space Shuttle and the Automated Transfer Vehicle (ATV) of the European Space Agency (ESA). The extreme environment of a hypersonic flow has a major impact on the design and analysis of the aerodynamic and thermal loading of a re-entry or hypersonic cruise vehicle. During a hypersonic flight, the species of the flow field are vibrationally excited, dissociated, and ionized because of the very strong shock wave which is created around a vehicle. ${ }^{1}$ Because of these phenomena, it is necessary to consider the flow to be in thermal and chemical nonequilibrium. For chemical nonequilibrium, concentrations of chemical species should be calculated by solving the mass conservation equation of each species. The energy equations of each energy mode must be considered to take into account the thermal nonequilibrium condition.

The basic governing equations of a hypersonic flow are given by Lee ${ }^{2}$ which are the mass conservation equations of the chemical species, the momentum conservation equations, and the energy equation for each energy mode. In order to consider chemical nonequilibrium, finite rate chemistry must be employed in the mass conservation equation for each species. The energy equations of each energy mode makes it possible to include thermal nonequilibrium.

The simulation of all energy modes, which are translational, rotational, vibrational, and electron energy, is quite complex and computationally very expensive. To reduce the computational complexity and cost, the two-temperature model is developed by Park. ${ }^{3}$ Park's model uses the translational temperature to characterize both the translational energy of species and the rotational energy of the molecules because the rotational temperature is quickly equilibrated with the translational temperature of heavy particles. The vibrational temperate deviates from the translational temperature because of the slow energy transfer between the vibrational and translational energy modes. The electron temperature also deviates from the translational temperature. Therefore, the two-temperature model uses a vibrational temperature to describe the vibrational energy of molecules and the electron energy.

However, separate analysis of the electron energy is important in some cases. In the analysis of radio blackout, the accurate prediction of electron density is also required because radio blackout is caused by high electron density. ${ }^{4}$ The electron number density can be affected by the electron temperature because it may influence the chemistry which is related to electrons such as electron-impact ionization and dissociation. In the present study, we introduce an electron temperature model including electron-vibrational relaxation. The relaxation time of the electron-vibrational energy coupling is calculated by using a proposed vibrational excitation rate coefficient model. In Sec. II, we describe a numerical model of the electron temperature including the Jacobian matrix of an electron energy equation. The details of the electron energy source terms are described in Sec. III. Section IV shows numerical results of several cases using the suggested 
electron temperature model. Conclusions are formulated in Sec. V.

\section{Electron Temperature Modeling}

A hypersonic flow can be described by the Navier-Stokes equations with thermochemical nonequilibrium effects which consist of the mass conservation equations of the chemical species, the momentum conservation equations, and the energy equation for each energy mode. The chemical nonequilibrium effect is considered using a finite rate chemistry model. ${ }^{5,6}$ A vibrational energy equation is employed to describe a vibrational temperature nonequilibrium. ${ }^{7}$

\section{II.A. Electron Energy Equation}

The electron energy equation that describes thermochemical nonequilibrium in a hypersonic flow was suggested by Lee: ${ }^{2}$

$$
\frac{\partial E_{e}}{\partial t}+\frac{\partial}{\partial x^{j}}\left[\left(E_{e}+p_{e}\right) u^{j}\right]-\frac{\partial}{\partial x^{j}}\left[-q_{e}^{j}+\tau_{e}^{i j} u^{i}-J_{e}^{j} e_{e}\right]=S_{e}
$$

where $E_{e}=\rho_{e}\left(C_{V, e} T_{e}+\frac{1}{2}\left(u^{2}+v^{2}+w^{2}\right)\right)$ is the electron energy, $e_{e}=\left(C_{V, e} T_{e}+\frac{1}{2}\left(u^{2}+v^{2}+w^{2}\right)\right)$ is the electron energy per unit mass of electrons, $\overrightarrow{q_{e}}$ is the heat flux vector of electrons, $\vec{J}_{e}$ is the diffusion flux vector of electrons, and $S_{e}$ is the source term. The electron energy source term, $S_{e}$, is given by

$$
S_{e}=S_{\text {trans-e }}+S_{\text {inelastic }, e}+S_{\text {chem }, e}
$$

where $S_{\text {trans-e }}$ is the energy transferred between translational and electron energy modes, $S_{\text {inelastic }-e}$ is the rate of inelastic energy exchange between electrons and molecules, and $S_{c h e m, e}$ is the electron energy gained by the electrons generated from chemical reactions.

Equation 1 has the similar form to a total energy equation. Compared to the vibrational or rotational energy equation, the electron energy equation has an additional term on the left-hand-side of Eq. 1, which is the electron pressure, $p_{e}$. Since the electron pressure term makes the Jacobian of the governing equations complicated, a modified form of the electron energy equation is required in order to reduce the computational cost in the calculation of the Jacobian. Therefore, the electron energy equation can be expressed as follows:

$$
\frac{\partial E_{e}}{\partial t}+\frac{\partial}{\partial x^{j}}\left[E_{e} u^{j}\right]-\frac{\partial}{\partial x^{j}}\left[-q_{e}^{j}+\tau_{e}^{i j} u^{i}-J_{e}^{j} e_{e}\right]=S_{e, \text { modified }}
$$

where $S_{e, \text { modified }}$ is the modified source term of the electron energy equation which includes the electron pressure term. The modified source term is given by

$$
S_{e, \text { modified }}=S_{\text {epg }}+S_{\text {trans-e }}+S_{\text {inelastic }, e}+S_{\text {chem, },}
$$

where $S_{\text {epg }}=-p_{e} \nabla \cdot \vec{u}$ is an approximation to the work done on electrons by the electric field induced by the electron pressure gradient. ${ }^{9}$

\section{II.B. Jacobian of Electron Energy Equation}

The inviscid flux vector with an electron energy is written as

$$
\vec{F}=\left[\begin{array}{c}
\rho_{1} U \\
\rho_{2} U \\
\vdots \\
\rho_{s} \\
\rho U u+p n_{x} \\
\rho U v+p n_{y} \\
\rho U w+p n_{z} \\
\rho U H \\
U E_{v i b} \\
U E_{e}
\end{array}\right]
$$


where $H$ is a total enthalpy per unit mass, $n_{x}, n_{y}$, and $n_{z}$ are the $\mathrm{x}, \mathrm{y}$, and $\mathrm{z}$ components of a unit vector normal to a computational cell face, and $U$ is the normal component of velocity through the cell face, $U=u n_{x}+v n_{y}+w n_{z} \cdot{ }^{9}$ The vector of conservative variables is given by

$$
\vec{Q}=\left[\begin{array}{c}
\rho_{1} \\
\rho_{2} \\
\vdots \\
\rho_{s} \\
\rho u \\
\rho v \\
\rho w \\
E \\
E_{v i b} \\
E_{e}
\end{array}\right]
$$

Pressure with an electron temperature is given by

$$
p=\sum_{s \neq \text { electron }}^{\text {All species }} \rho_{s} \frac{\bar{R}}{M_{s}} T+\rho_{e} \frac{\bar{R}}{M_{e}} T_{e}
$$

where $M_{s}$ is a species molecular weight. Therefore, the differential form for pressure can be described as

$$
d p=T \sum_{s \neq \text { electron }}^{\text {All species }} d \rho_{s} \frac{\bar{R}}{M_{s}}+d T \sum_{s \neq \text { electron }}^{\text {All species }} \rho_{s} \frac{\bar{R}}{M_{s}}+d \rho_{e} \frac{\bar{R}}{M_{e}} T_{e}+\rho_{e} \frac{\bar{R}}{M_{e}} d T_{e}
$$

The derivatives of an electron temperature for conserved variables can be written

$$
\begin{gathered}
\frac{\partial T_{e}}{\partial \rho_{s}}=\frac{\frac{\rho_{e}}{\rho}\left(u^{2}+v^{2}+w^{2}\right)}{\rho_{e} C_{v, e}} \\
\frac{\partial T_{e}}{\partial \rho_{e}}=\frac{\left(\frac{\rho_{e}}{\rho} \frac{1}{2}\right)\left(u^{2}+v^{2}+w^{2}\right)}{\rho_{e} C_{v, e}} \\
\frac{\partial T_{e}}{\partial \rho u}=\frac{-\frac{\rho_{e}}{\rho} u}{\rho_{e} C_{v, e}} \\
\frac{\partial T_{e}}{\partial E}=0 \\
\frac{\partial T_{e}}{\partial E_{v i b}}=0 \\
\frac{\partial T_{e}}{\partial E_{e}}=\frac{1}{\rho_{e} C_{v, e}}
\end{gathered}
$$

where $s$ is a species except an electron.

The Jacobian matrix of inviscid fluxes, Eq. 5, is listed in Appendix A. For an implicit method, the electron energy expression using the electron energy per unit mass, $E_{e}=\rho_{e} e_{e}$, is difficult to be employed in the calculation of the Jacobians, because the derivative of the electron energy for species except electrons, $\frac{\partial E_{e}}{\partial \rho_{e}}$, becomes zero. The zero derivatives cause a singularity problem in the Jacobian matrix. To prevent the singularity of the Jacobian matrix, the electron energy can be expressed as follows:

$$
E_{e}=\rho \varepsilon_{e}
$$

where $e_{e}$ is modified electron energy per unit mass, $e_{e}=\frac{\rho_{e}}{\rho} \varepsilon_{e}$. Using modified electron energy per unit mass, $\dot{e}_{e}$, left and right eigenvectors of the Jacobian can be obtained as described in Appendix B.

\section{4 of 22}




\section{II.C. Chemistry}

The chemical reaction rates are functions of different mode temperatures, depending on the type of reactions. Using Park's two temperature model, the controlling temperature, $T_{c}$, is given by: ${ }^{3}$

$$
T_{c}=T_{t r}^{a} T_{v e}^{b}
$$

where the parameters $\mathrm{a}$ and $\mathrm{b}$ indicate the importance of each temperature mode on a specific chemical reaction. When an electron temperature model is considered, Eq. 16 should be modified as follows:

$$
T_{c}=T_{t r}^{a} T_{v i b}^{\dot{b}} T_{e}^{c^{c}}
$$

The values of $\dot{a}, \hat{b}$, and $\dot{c}$ used in an electron temperature model are listed in Table 1 in terms of reaction type. $^{10}$

Table 1. Coefficientss of the controlling temperature with an electron temperature model for various reaction types

\begin{tabular}{lcccccc}
\hline \hline \multirow{2}{*}{ Reaction } & \multicolumn{3}{c}{ Forward } & \multicolumn{4}{c}{ Backward } \\
& $\dot{a}_{f}$ & $\dot{b}_{f}$ & $\dot{b}_{f}$ & $\dot{a}_{b}$ & $\dot{b}_{b}$ & $\dot{c}_{b}$ \\
\hline Dissociation & 0.67 & 0.33 & 0.0 & 1.0 & 0.0 & 0.0 \\
Neutral exchange & 1.0 & 0.0 & 0.0 & 1.0 & 0.0 & 0.0 \\
Associative ionization & 1.0 & 0.0 & 0.0 & 0.0 & 0.5 & 0.5 \\
Charge exchange & 1.0 & 0.0 & 0.0 & 1.0 & 0.0 & 0.0 \\
Electron impact ionization & 0.0 & 0.0 & 1.0 & 0.0 & 0.0 & 1.0 \\
\hline
\end{tabular}

\section{Electron Energy Source Terms}

As shown in Eq. 4, the electron energy source term consists of electron pressure, elastic collision, inelastic collision and chemistry related terms which are $S_{\text {epg }}, S_{\text {trans-e }}, S_{\text {inelastic,e }}$, and $S_{\text {chem,e }, e}$, respectively. The elastic collision term, $S_{\text {trans-e }}$, indicates the energy relaxation between translational and electron energies by electron collisions. The chemistry related term, $S_{c h e m, e}$, gives added or removed electron energy by chemical reactions and can be expressed as:

$$
S_{\text {chem,e }}=\dot{\omega}_{e} \varepsilon_{e}
$$

The inelastic energy exchange term, $S_{\text {inelastic,e }}$, includes rotational-electron relaxation, electron-vibrational relaxation, radiation loss, and an electron-impact ionization term. Therefore, the rate of inelastic energy exchange between electrons and molecules,$S_{\text {inelastic, },}$, is given by: ${ }^{11}$

$$
S_{\text {inelastic }, e}=S_{\text {rot-e }}-S_{e-v}-S_{\text {rad }}-\sum_{f}^{\text {all electron-impact-ionization }}\left[\dot{\omega}_{f, e} E_{i, f}\right]
$$

where $E_{i, f}$ is the first ionization energy. Last term of Eq.19, $\sum_{f}^{\text {all electron-impact-ionization }}\left[\dot{\omega}_{f, e} E_{i, f}\right]$, indicates an electron energy removed by an electron-impact ionization.

\section{III.A. Translational-Electron Relaxation}

The energy transfer between the translational energy of heavy particles and the electron energy, $S_{\text {trans-e }}$, is given by

$$
S_{\text {tran }-e}=2 \rho_{e} \frac{3}{2} k\left(T_{\text {trans }}-T_{e}\right){\sqrt{\frac{8 k T_{e}}{\pi m_{e}}}}^{\text {All }} \sum_{\text {sfelectron }}^{\text {species }}\left(\frac{\rho_{s}}{m_{s}^{2}} \sigma_{e s}\right)
$$


where $\sigma_{e s}$ is the collision cross section between electrons and $s$ species. For electron-ion collisions, the Coulomb collision can be employed to estimate the cross section, $\sigma_{e s}{ }^{12}$

$$
\sigma_{e s}=\frac{4}{3} 2 \pi b_{0}^{2} \ln \left[1+{\frac{\lambda_{D}}{b_{0}}}^{2}\right]
$$

where $b_{0}=\frac{Z e^{2}}{12 \pi \varepsilon_{0} k T_{e}}\left[m^{2}\right]$ is a scattering parameter for $90^{\circ}$ and $\lambda_{D}=\left(\frac{\varepsilon_{0} k T_{e}}{e^{2} n_{e}}\right)^{1 / 2}\left[m^{2}\right]$ is the Debye length. For electron-neutral collisions, the collision cross section is expressed by the following approximation: ${ }^{9}$

$$
\sigma_{e s}=a_{s}+b_{s} T_{e}+c_{s} T_{e}^{2}
$$

where $a_{s}, b_{s}$ and $c_{s}$ are coefficients. The values of coefficients, $a_{s}, b_{s}$ and $c_{s}$, are listed in Table 2 for several neutrals. $^{13}$

Table 2. The coefficients of the electron-neutral collisional cross section

\begin{tabular}{cccc}
\hline \hline Species & $a_{s}$ & $b_{s}$ & $c_{s}$ \\
\hline$N_{2}$ & $7.5 \times 10^{-20}$ & $5.5 \times 10^{-24}$ & $-1.0 \times 10^{-28}$ \\
$O_{2}$ & $2.0 \times 10^{-20}$ & $6.0 \times 10^{-24}$ & 0.0 \\
$N O$ & $1.0 \times 10^{-19}$ & 0.0 & 0.0 \\
$N$ & $5.0 \times 10^{-20}$ & 0.0 & 0.0 \\
$O$ & $1.2 \times 10^{-20}$ & $1.7 \times 10^{-24}$ & $-2.0 \times 10^{-29}$ \\
\hline
\end{tabular}

\section{III.B. Rotational-Electron Relaxation}

The energy relaxation between the rotational and electron energies should be considered in the electron energy equation because of the electron interactions with the molecular multipoles. In order to simplify the relaxation term, an energy transfer rate factor, $g_{r o t, s}$, can be employed in the expression of the rotationalelectron energy transfer rate. ${ }^{13}$ The rate factor is the ratio of the rotational-electron energy relaxation time to the translational-electron energy relaxation time for the molecular species. Therefore, the relaxation between rotational and electron energies can be expressed in the following form:

$$
S_{\text {rot }, e}=2 \rho_{e} \frac{3}{2} k\left(T_{\text {trans }}-T_{e}\right) \sqrt{\frac{8 k T_{e}}{\pi m_{e}}} \sum_{s \neq \text { electron }}^{\text {All molecules }}\left(g_{\text {rot }, s} \frac{\rho_{s}}{m_{s}^{2}} \sigma_{e s}\right)
$$

The rotational excitation by electrons in homonuclear diatomic molecules such as $N_{2}$ and $O_{2}$ is mainly due to the quadrupole or induced polarization interaction. For a heteronuclear case such as $N O$, the main cause of the excitation is a permanent electric dipole moment. Since the permanent electric dipole moment is more efficient for the rotational excitation than the quadrupole moment, a heteronuclear molecule should have a much higher value of the relaxation rate factor than a homonuclear molecule. For neutral species, the energy transfer rate factor, $g_{r o t, s}$, is listed as Table 3. For molecular ions, we assume they have the same rate factor as their neutral molecules.

Table 3. The energy transfer rate factors of rotational-electron energy relaxation

\begin{tabular}{ccl}
\hline \hline Species & $g_{\text {rot }}$ & Reference \\
\hline$N_{2}$ & 10 & 14 \\
$O_{2}$ & 10 & 15 \\
$N O$ & 100 & 16 \\
\hline
\end{tabular}




\section{III.C. Vibrational-Electron Relaxation}

The rate of energy transfer between electron and vibrational energies, $S_{e-v}$, is assumed to be of the LandauTeller form: ${ }^{6}$

$$
Q_{e-v, s}=\rho_{s} \frac{e_{v i b, s}^{*}\left(T_{e}\right)-e_{v i b, s}(T)}{\tau_{e s}}
$$

where $\tau_{e s}$ is the electron-vibrational relaxation time. In the present study, only the vibrational-electron energy coupling of diatomic nitrogen is considered because the coupling of other molecules such as $\mathrm{O}_{2}$ and $N O$ is two orders of magnitude weaker. ${ }^{17}$

For diatomic nitrogen, the relaxation time is derived by Lee: ${ }^{18}$

$$
\tau_{e s}=\frac{k T_{e}}{p_{e} \cdot\left[\left(1-e^{-\theta_{v} / T_{e}}\right)^{2} \cdot \frac{1}{2} \int k_{0, j}^{e-v} j^{2} \mathrm{~d} j\right]}
$$

where $k_{0, j}$ is a vibrational excitation rate coefficient from vibrational state 0 to $j$. The vibrational excitation rate coefficient of $N_{2}$ by electrons was previously modeled by Bourdon et al. ${ }^{19}$ as follows:

$$
k_{0-j}^{e-v}\left(T_{e}\right)=10^{-15}\left(\frac{a}{x}+b+c x+d x^{2}\right) x^{3 / 2} \cdot \exp \left(\frac{f}{x}\right)
$$

where $x=\frac{T_{e}}{10000}$.

However, the previous rate coefficient model is not applicable to calculate the electron-vibrational relaxation time below $2,000 \mathrm{~K}$ or above $50,000 \mathrm{~K}$ because it gives a negative value of the vibrational excitation rate coefficient. Therefore, we propose a vibrational excitation rate coefficient model to obtain the relaxation time over the low or high temperature regimes as follows:

$$
k_{0-j}^{e-v}\left(T_{e}\right)=10^{-15} \cdot \dot{a} T_{e}^{3 / 2} \cdot \exp \left(\frac{\dot{b}}{T_{e}}+\dot{c}\right)
$$

where $T_{e}$ is in eV. The coefficients $a, b, b$, and $\dot{c}$ in Eq. 27 are listed in Table 4.

Table 4. The coefficients of the vibrational excitation rate coefficient model

\begin{tabular}{cccc}
\hline \hline$j$ & $\dot{a}$ & $\dot{b}$ & $\dot{c}$ \\
\hline 1 & 8.034 & -2.227 & 2.005 \\
2 & 7.924 & -2.235 & 1.479 \\
3 & 7.876 & -2.257 & 1.054 \\
4 & 7.626 & -2.334 & 0.6499 \\
5 & 7.326 & -2.454 & 0.2049 \\
6 & 4.900 & -2.556 & 0.007448 \\
7 & 2.457 & -2.702 & 0.002952 \\
8 & 1.119 & -2.865 & 0.001133 \\
9 & 0.4681 & -3.042 & 0.004312 \\
10 & 0.1837 & -3.223 & 0.0002219 \\
\hline
\end{tabular}

Figure 1 shows the calculated vibrational rate coefficient using different models compared with experimental data. As can be seen, the proposed model gives a better match of the rate coefficient with the theoretical and experimental data and it does not produce a negative value at high temperature.

Figure 2 shows the calculated electron-vibrational relaxation time using the proposed vibrational rate coefficient model for $e-N_{2}$ collision compared with Lee's data. ${ }^{18}$ As can be seen, the model agrees well with Lee's theoretical data and makes it possible to calculate the relaxation time in low temperature and high temperature regimes. In the calculation of the relaxation time, we assume that the contribution of higher 
energy transitions is negligible. Therefore, the integration of the second moment of the rate coefficient can be simplified as follows:

$$
\int k_{0, j}^{e-v} j^{2} \mathrm{~d} j \approx \sum_{j=1}^{10} k_{0, j}^{e-v} j^{2}
$$

\section{Numerical Results}

The electron temperature model is numerically simulated with an implicit method using a hypersonic flow code. The implemented electron temperature model is applied to different flow conditions which are a cylinder flow, a RAM-C test case, an ATV case, and a Stardust case. In the simulation, it is initially assumed that the flow has a very small level of electron density, which is $10^{-12}$ by mass of the mixture density because electrons are required in order to calculate the electron temperature.

\section{IV.A. Cylinder Flow}

For a ground-based experiment of hypersonic flow, an arc heated plasma wind tunnel is usually employed because it generates a similar flow environment which a vehicle experiences during hypersonic flight. Inside of a plasma wind tunnel, the injected working gas becomes high enthalpy flow by arc heating, and it is accelerated through a nozzle up to hypersonic velocity. Inside of a plasma wind tunnel, the generated flow is usually in a very strong thermal nonequilibrium state of electron temperature. ${ }^{20}$ Therefore, a cylinder flow in a plasma wind tunnel is a useful validation case of the electron temperature model.

In this case, the freestream is composed of $\mathrm{Ar}, \mathrm{Ar}^{+}$, and electrons and freestream conditions are given in Table 5. Figures 3 and 4 show the simulation result of a cylinder flow in argon with the electron temperature model which has significant nonequilibrium of the translational and electron inflow temperatures. The significant nonequilibrium state of the translational and electron temperatures can verify the capability of the electron temperature model. As can be seen, the electron temperature is almost frozen and the flow has significant thermal nonequilibrium between the translational and electron energy modes. Behind the shock, the measured electron temperature is about $6500 \pm 1500 \mathrm{~K}^{21}$ which agrees well with the simulated result.

Table 5. Freestream condition of a cylinder flow

\begin{tabular}{cccccc}
\hline \hline$V_{\infty}[\mathrm{m} / \mathrm{s}]$ & $\rho_{A r}\left[\mathrm{~kg} / \mathrm{m}^{3}\right]$ & $\rho_{A r^{+}}\left[\mathrm{kg} / \mathrm{m}^{3}\right]$ & $e^{-}\left[\mathrm{kg} / \mathrm{m}^{3}\right]$ & $T_{\infty}[\mathrm{K}]$ & $T_{e, \infty}[\mathrm{K}]$ \\
\hline 2150 & $1.187 \times 10^{-4}$ & $1.875 \times 10^{-8}$ & $2.551 \times 10^{-13}$ & 180 & 6000 \\
\hline
\end{tabular}

\section{IV.B. RAM-C}

The Radio Attenuation Measurement (RAM) program was a series of hypersonic experiments performed in the 1960's to study communication blackout. In order to assess the electron temperature model in a multi-species chemistry flow, the RAM-C test case is simulated at $61 \mathrm{~km}$ altitude condition with a 7 -species air chemistry model including electrons. The geometry of the vehicle is a sphere-cone of $0.1524 \mathrm{~m}$ radius with a cone half-angle of 9 degree. ${ }^{22}$ The details of the flow conditions at altitude of $61 \mathrm{~km}$ are listed in Table 6 .

Table 6. Flow conditions for the RAM-C simulation at $61 \mathrm{~km}$ altitude

\begin{tabular}{cccc}
\hline \hline Mach number & $T_{\infty}[K]$ & $T_{\infty, \text { wall }}[K]$ & $P_{\infty}[\mathrm{Pa}]$ \\
\hline 23.9 & 254 & 1500 & 19.7 \\
\hline
\end{tabular}

Figures 5-7 show the contours of each temperature mode for the RAM-C test case at $61 \mathrm{~km}$ altitude. The maximum electron temperature downstream of the shock is about 9,000 K which is slightly lower than the maximum vibrational temperature. Temperature distributions along the stagnation streamline are presented in Fig. 8. As can be seen, the translational temperature reaches a peak of about 22,000 K then decreases towards the wall temperature, which is $1,500 \mathrm{~K}$. The electron temperature is similar to the vibrational 
temperature near the wall and becomes lower than the vibrational temperature near the shock. Near the shock region, the vibrational and electron temperatures are in a strong thermal nonequilibrium state. Since the electron temperature is related to electron-impact ionization, the electron temperature model should be considered to accurately predict electron number densities for this type of vehicle.

\section{IV.C. ATV}

The automated transfer vehicle (ATV) is a cylinder shaped reentry vehicle with a diameter of $4.5 \mathrm{~m}^{23}$ which is a supply vehicle for the International Space Station (ISS). The ATV is simulated at a $75 \mathrm{~km}$ altitude reentry condition, and the details of the freestream conditions are listed in Table.

Table 7. Flow conditions for the ATV simulation at $75 \mathrm{~km}$ altitude

\begin{tabular}{ccccc}
\hline \hline$V_{\infty}[\mathrm{m} / \mathrm{s}]$ & $T_{\infty}[K]$ & $T_{\infty, \text { wall }}[\mathrm{K}]$ & $\rho_{N_{2}, \infty}\left[\mathrm{kg} / \mathrm{m}^{3}\right]$ & $\rho_{\mathrm{O}_{2}, \infty}\left[\mathrm{kg} / \mathrm{m}^{3}\right]$ \\
\hline 7330 & 224 & 1600 & $2.17 \times 10^{-5}$ & $6.62 \times 10^{-6}$ \\
\hline
\end{tabular}

Figures 9-11 show the results for ATV case with an 11-species air chemistry model which includes electronimpact ionization reactions. The maximum electron temperature downstream of the shock is about $12,000 \mathrm{~K}$ which is slightly lower than the maximum vibrational temperature, 10,000 K. A profile along the stagnation streamline of temperatures is presented in Fig. 12. As can be seen, the electron temperature is slightly higher than the translational temperature near the shock because the electron density is very small in front of the shock. Because of the diffusion of electrons, the electron temperature becomes higher than the translational temperature in front of the shock. However, the flow is close to thermal equilibrium behind the shock.

\section{IV.D. Stardust}

The Stardust payload was launched in 1999 on a mission to collect samples from the interstellar dust. A previous DSMC Stardust simulation showed significant nonequilibrium of the electron temperature, but there is no prior CFD analysis of Stardust that includes the electron temperature. Therefore, reentry of Stardust is simulated at the $64.7 \mathrm{~km}$ altitude condition using the electron temperature model. The employed freestream condition is given in Table 8 .

Table 8. Freestream conditions of Stardust entry capsule at $64.7 \mathbf{~ k m ~ a l t i t u d e}{ }^{24}$

\begin{tabular}{ccccc}
\hline \hline Altitude $[\mathrm{km}]$ & Velocity $[\mathrm{m} / \mathrm{s}]$ & Density $\left[\mathrm{kg} / \mathrm{m}^{3}\right]$ & Temperature $[\mathrm{K}]$ & Pressure $[\mathrm{Pa}]$ \\
\hline 64.7 & 11400 & $1.41 \times 10^{-4}$ & 231 & 9.33 \\
\hline
\end{tabular}

Figures 13-15 show the results for the Stardust simulation with an 11-species air chemistry model which includes two electron-impact ionization reactions. The numerical results indicate the flow is in a significant thermal nonequilibrium state. The detailed profiles along the stagnation streamline of temperatures are presented in Fig. 16. As can be seen, electron temperature is in a very strong nonequilibrium state. It is clear that the structure of the temperature distributions is different than that of RAM-C because Stardust

has a higher reentry velocity and the bluntness of the vehicle is increased. Similar to the ATV case, Stardust also has a regime in which the electron temperature is slightly higher than the translational temperature. It means the diffusion of electrons is very important near the shock when the flow is in a strong thermal nonequilibrium state.

\section{Conclusions}

Electron temperature plays an important role in the study of Thermal Protection System (TPS) and radio blackout during a hypersonic flight, because it is related to the electron-impact ionization. Therefore, an electron energy equation should be considered whenever flows are in a strong thermal nonequilibrium state. In this study, the electron energy relaxations of each energy mode are accounted for which include translational-electron, rotational-electron, and vibrational-electron energy relaxation. In order to reduce the computational expense, only the vibrational-electron energy coupling of diatomic nitrogen is considered because the relaxation time of other molecules such as $\mathrm{O}_{2}$ and $\mathrm{NO}$ is two orders of magnitude higher. Solving 
a separate electron energy equation is computationally very difficult and expensive because of the singularity of the Jacobian matrix. The singularity problem is removed by introducing a modified electron energy term, Eq. 15. Since the modified electron energy expression prevents the zero derivative of electron energy, it is useful to calculate the Jacobian for an implicit method.

The electron temperature model is implemented into a hypersonic flow code for both explicit and implicit methods. In the present study, we simulated several cases which are a cylinder flow, a RAM-C case, the entry of the ATV, and Stardust reentry capsule in order to assess the electron temperature model. The simulation results show that the electron temperature model can simulate significant nonequilibrium of the translational and electron temperatures. Near the shock regime, the electron temperature is slightly higher than the translational temperature because of the diffusion of electrons. The calculation of electron temperature using continuum simulation techniques (CFD) is unique in the simulation of the Stardust reentry capsule which can represent a strong thermal nonequilibrium condition. Therefore, the electron temperature model of the present study can be employed to simulate a significant thermal nonequilibrium state of electron temperature with reasonable computational effort. 


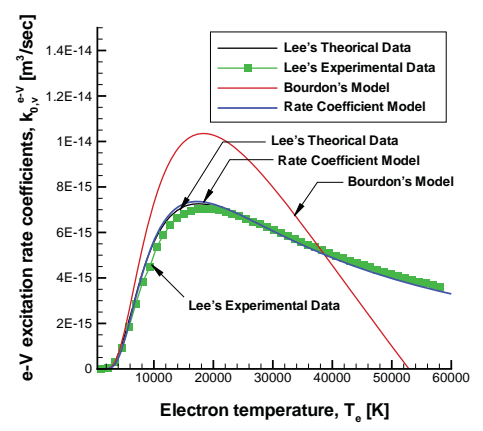

(a)

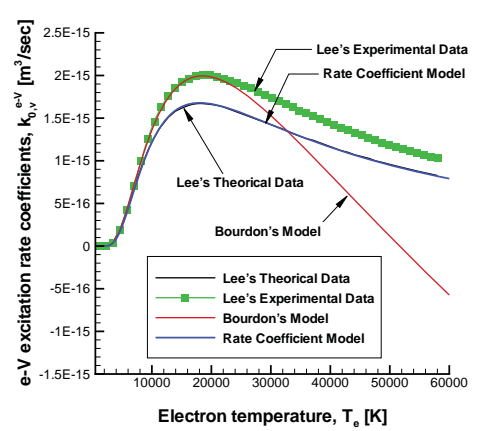

(d)

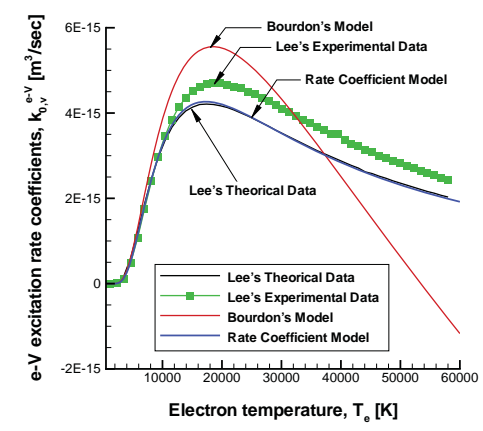

(b)

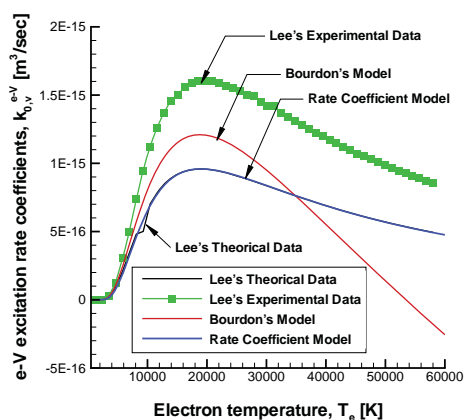

(e)

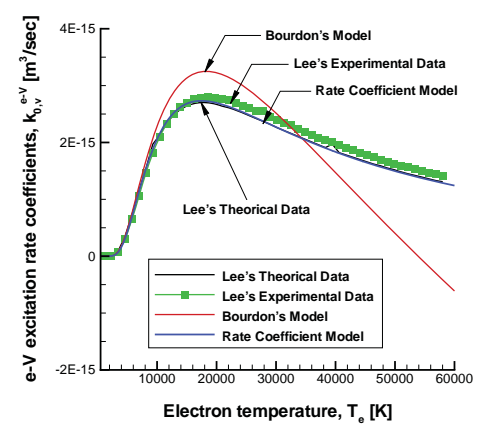

(c)

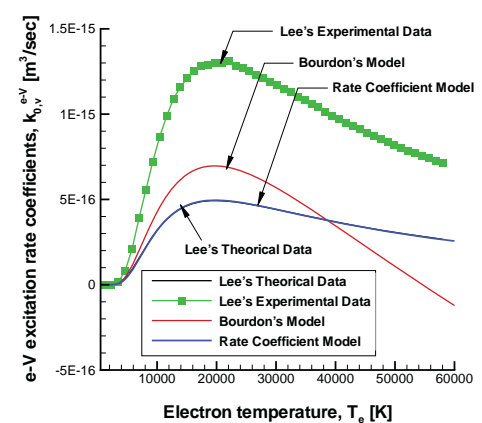

(f)

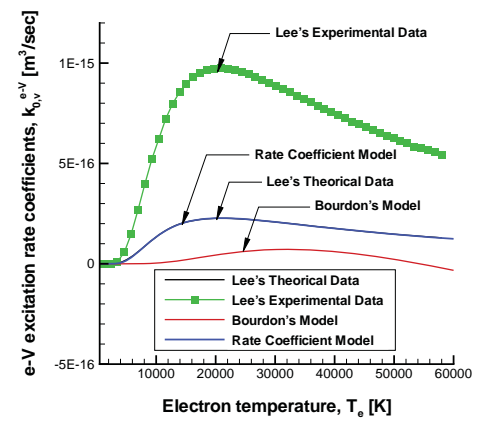

(g)

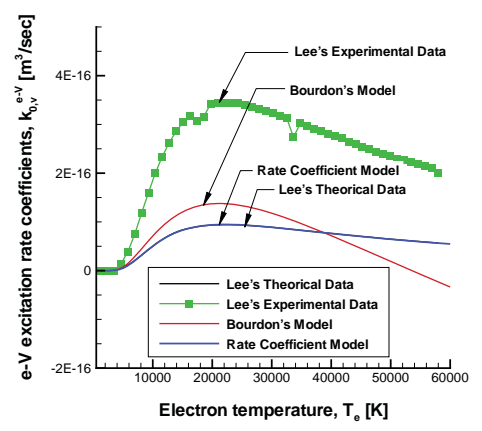

(h)

Figure 1. Electron-vibration excitation rate coefficient, $k_{0, v}^{e-V}\left[\mathrm{~m}^{3} / \mathrm{sec}\right]:(\mathrm{a}) \mathbf{v}=\mathbf{1}(\mathrm{b}) \mathbf{v}=\mathbf{2}(\mathrm{c}) \mathbf{v}=\mathbf{3}(\mathrm{d}) \mathbf{v}=\mathbf{4}(\mathrm{e}) \mathbf{v}=\mathbf{5}(\mathrm{f})$ $\mathrm{v}=6(\mathrm{~g}) \mathrm{v}=\mathbf{7}(\mathrm{h}) \mathrm{v}=\mathbf{8}$ 


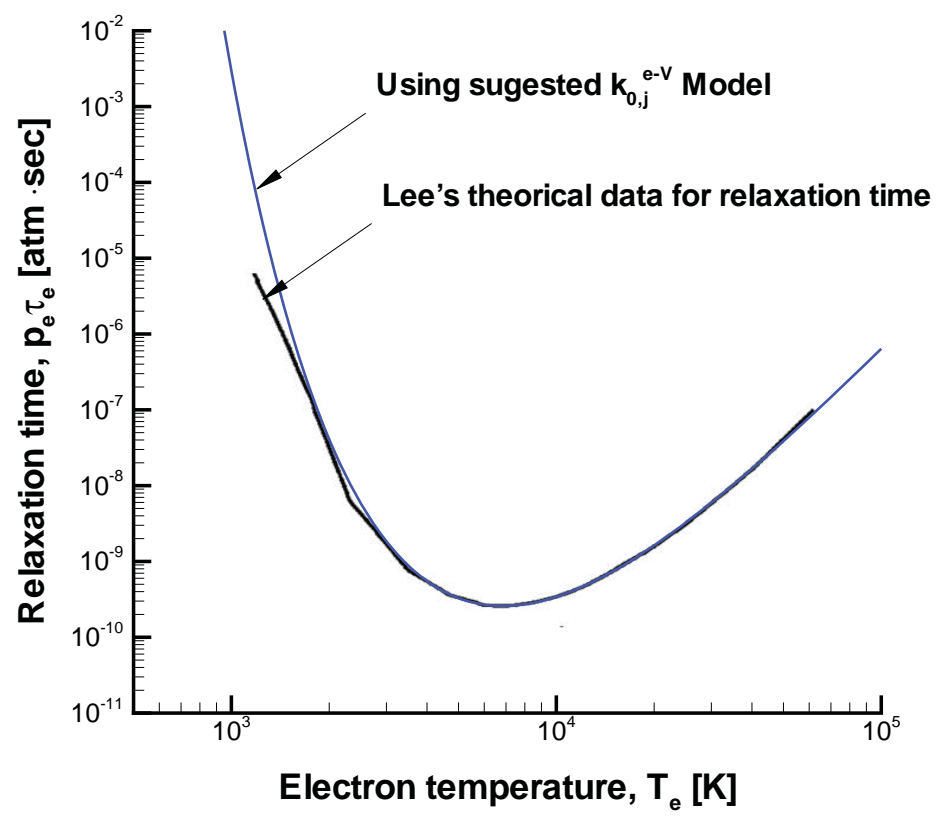

Figure 2. Vibrational relaxation time for $e-N_{2}$ collision using the proposed vibrational excitation rate coefficient model compared with Lee's relaxation time for $N_{2} \cdot{ }^{18}$

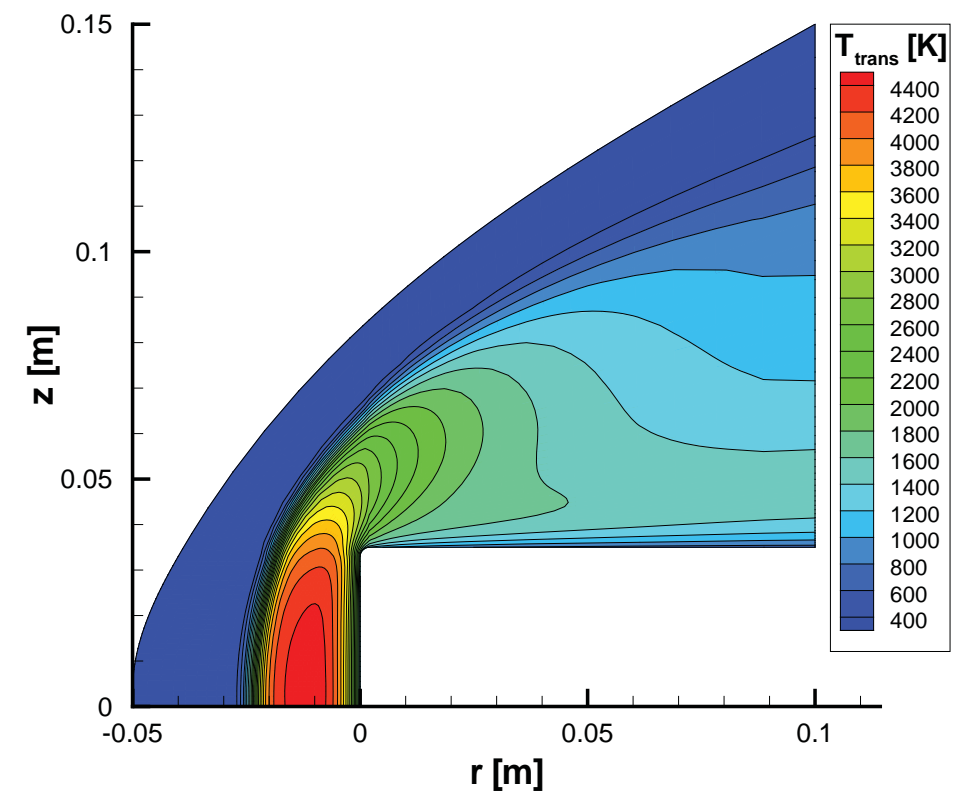

Figure 3. Translational temperature contours of the cylinder flow in argon; $V_{\infty}=2150[\mathrm{~m} / \mathrm{s}] ; T_{\infty}=180[\mathrm{~K}] ; T_{e, \infty}=$ $6000[K]$ 


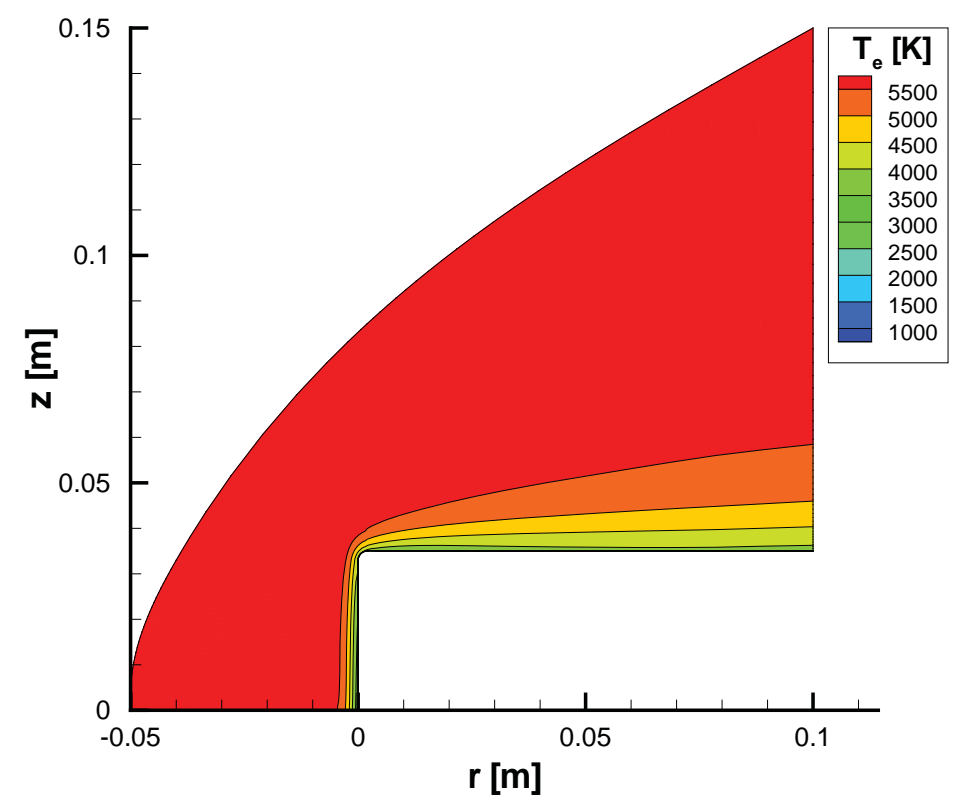

Figure 4. Electron temperature contours of the cylinder flow in argon; $V_{\infty}=2150[\mathrm{~m} / \mathrm{s}] ; T_{t r, \infty}=180[\mathrm{~K}] ; T_{e, \infty}=6000[\mathrm{~K}]$

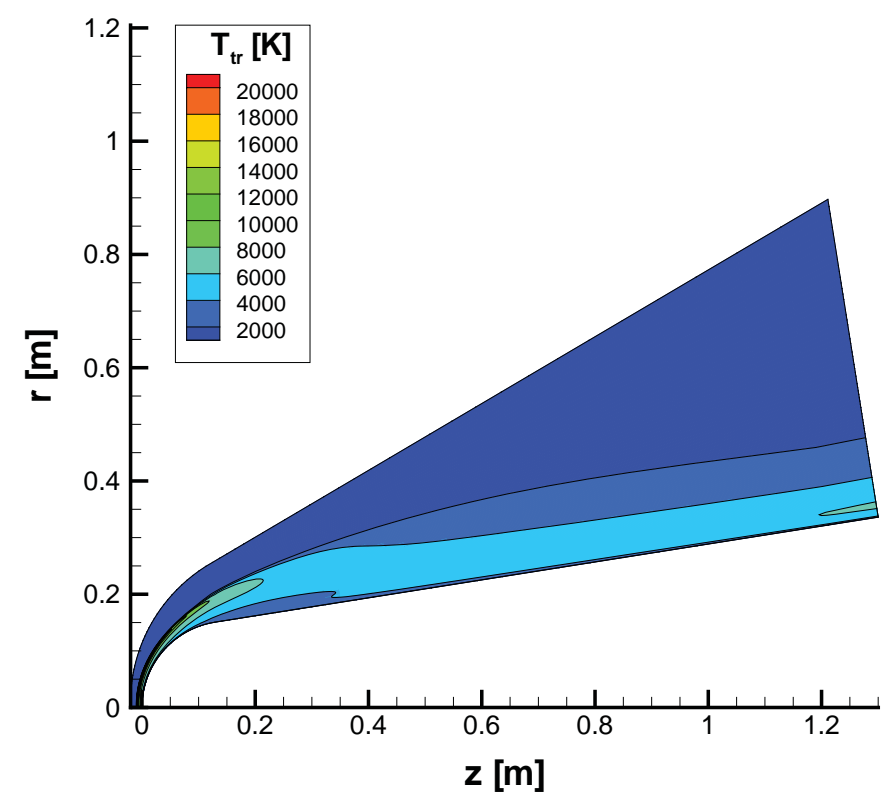

Figure 5. Translational temperature contours of the RAM-C at $61 \mathrm{~km} ; M_{\infty}=23.9$ 


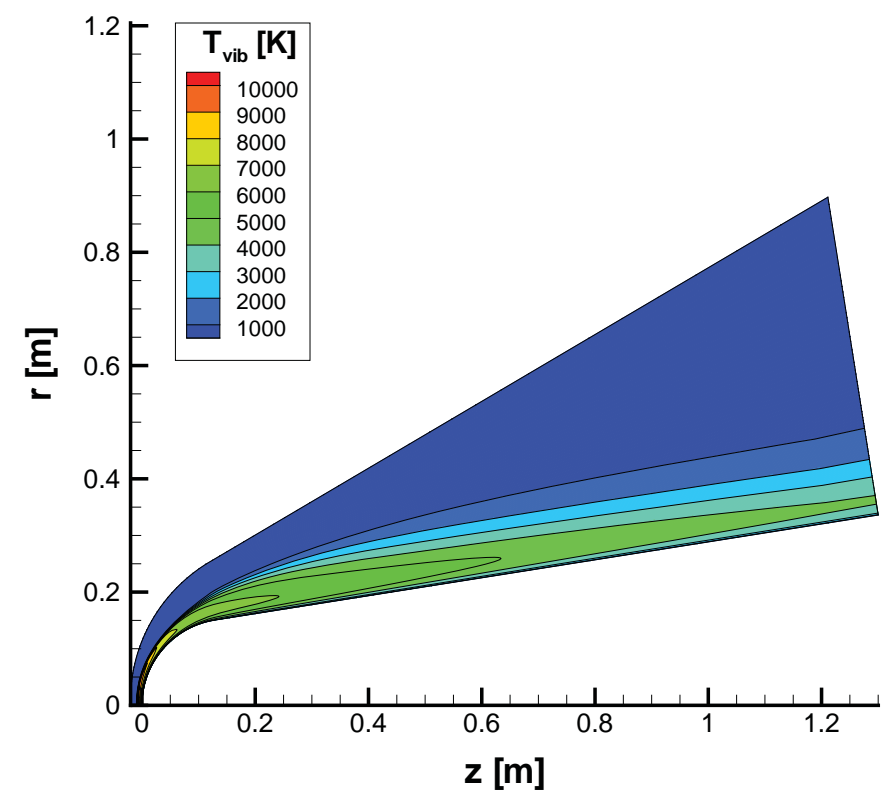

Figure 6. Vibrational temperature contours of the RAM-C at $\mathbf{6 1} \mathbf{k m} ; M_{\infty}=23.9$

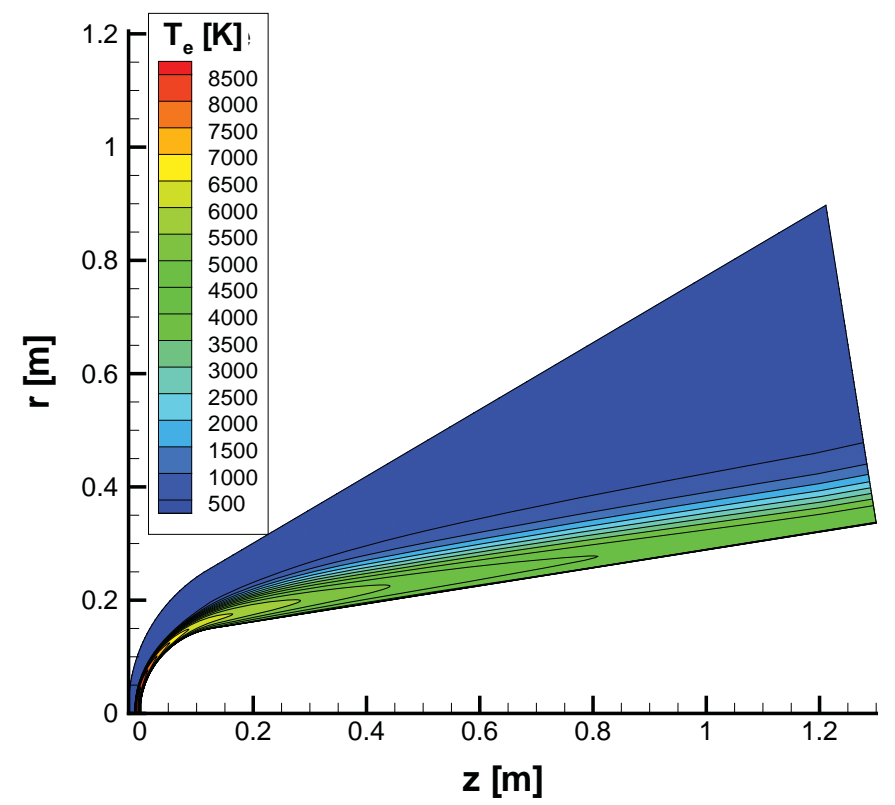

Figure 7. Electron temperature contours of the RAM-C at $61 \mathrm{~km} ; M_{\infty}=23.9$ 


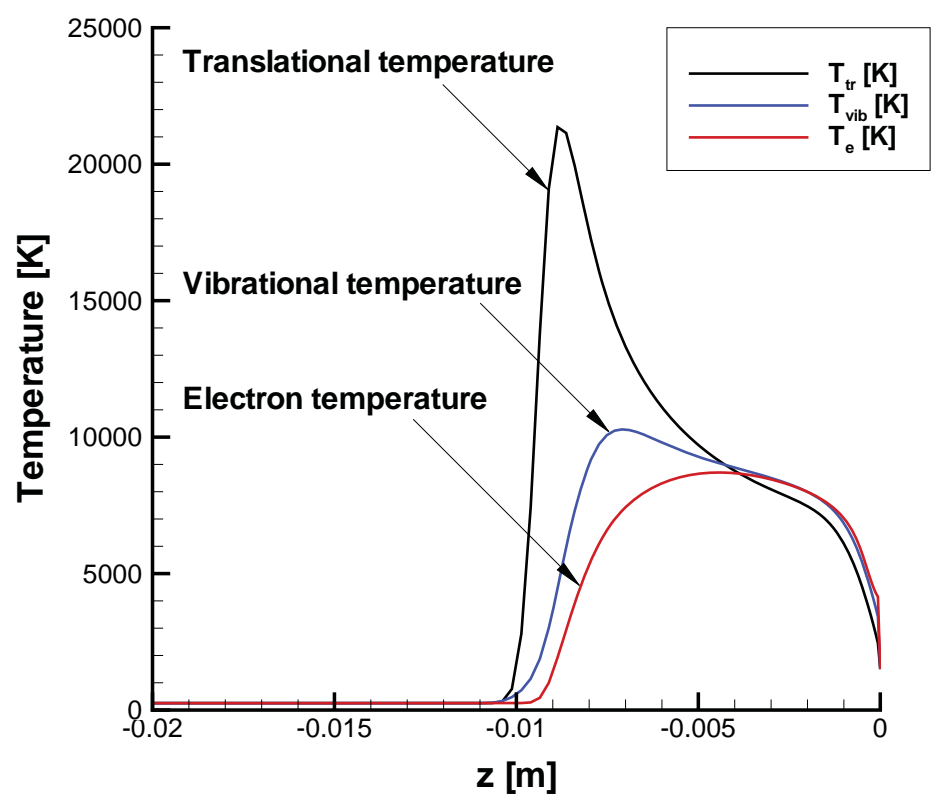

Figure 8. Temperature distributions along stagnation streamline of RAM-C at $\mathbf{6 1} \mathbf{k m}$ altitude; $M_{\infty}=23.9$

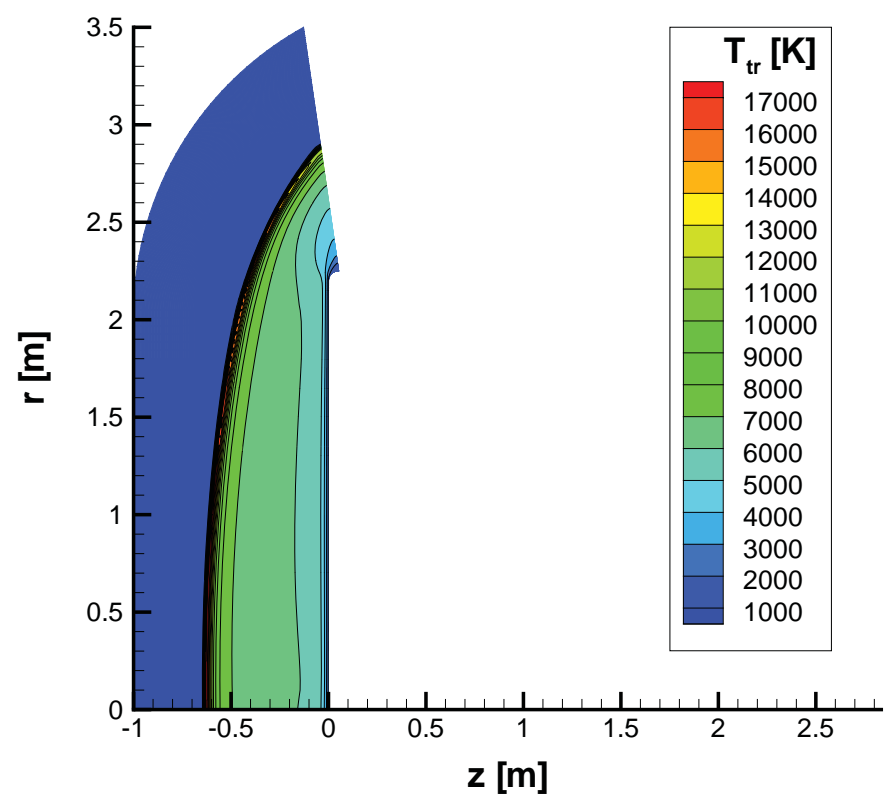

Figure 9. Translational temperature contours of the ATV; $V_{\infty}=7329[\mathrm{~m} / \mathrm{s}] ; \rho_{N_{2}, \infty}=2.1667 \times 10^{-5}\left[\mathrm{~kg} / \mathrm{m}^{-3}\right] ; \rho_{\mathrm{O}_{2}, \infty}=$ $6.6157 \times 10^{-6}\left[\mathrm{~kg} / \mathrm{m}^{-3}\right] ; T_{\infty}=223.9[\mathrm{~K}] ; T_{\text {wall }}=1600[\mathrm{~K}]$ 


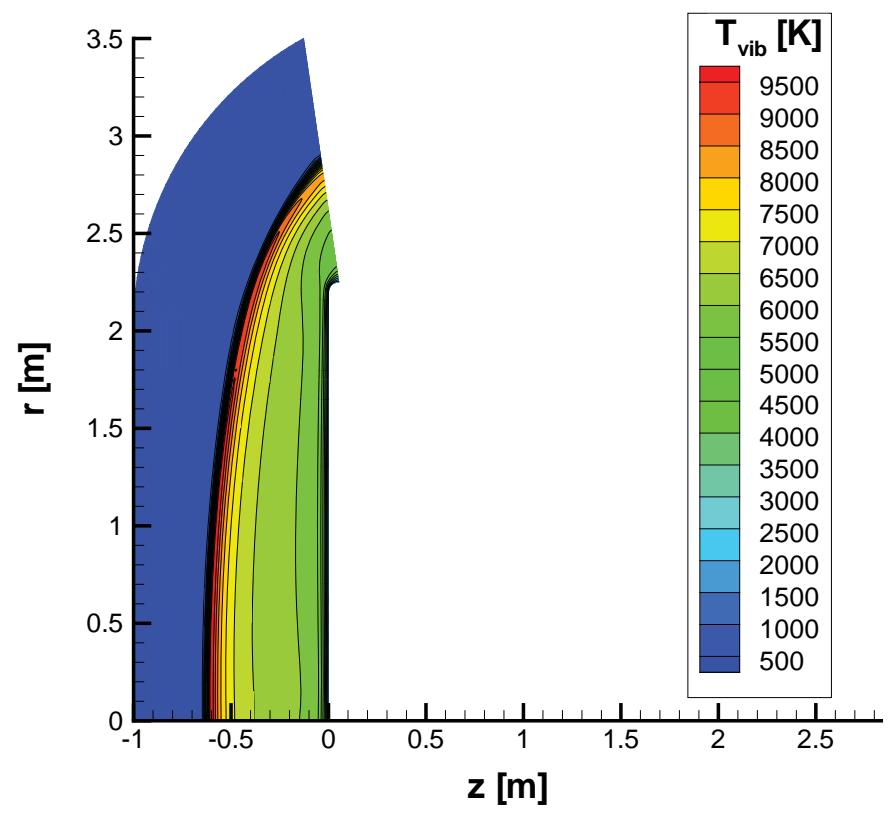

Figure 10. vibrational temperature contours of the ATV; $V_{\infty}=7329[\mathrm{~m} / \mathrm{s}] ; \rho_{N_{2}, \infty}=2.1667 \times 10^{-5}\left[\mathrm{~kg} / \mathrm{m}^{3}\right] ; \rho_{\mathrm{O}_{2}, \infty}=$ $6.6157 \times 10^{-6}\left[\mathrm{~kg} / \mathrm{m}^{3}\right] ; T_{\infty}=223.9[\mathrm{~K}] ; T_{\text {wall }}=1600[\mathrm{~K}]$

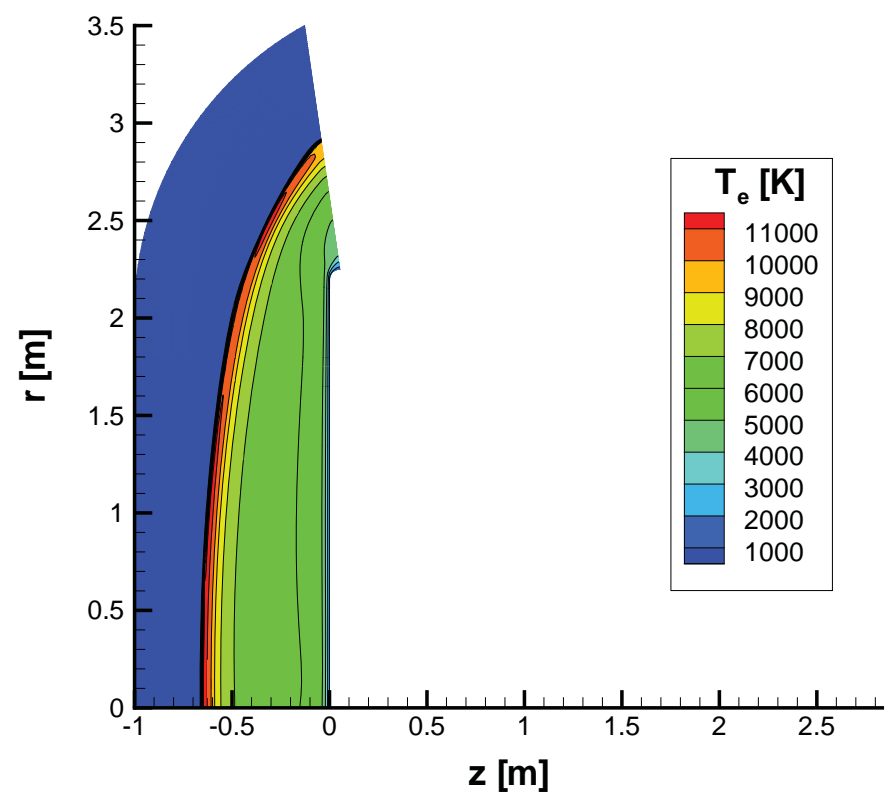

Figure 11. Electron temperature contours of the ATV; $V_{\infty}=7329[\mathrm{~m} / \mathrm{s}] ; \rho_{N_{2}, \infty}=2.1667 \times 10^{-5}\left[\mathrm{~kg} / \mathrm{m}^{3}\right] ; \rho_{\mathrm{O}_{2}, \infty}=$ $6.6157 \times 10^{-6}\left[\mathrm{~kg} / \mathrm{m}^{3}\right] ; T_{\infty}=223.9[\mathrm{~K}] ; T_{\text {wall }}=1600[\mathrm{~K}]$ 


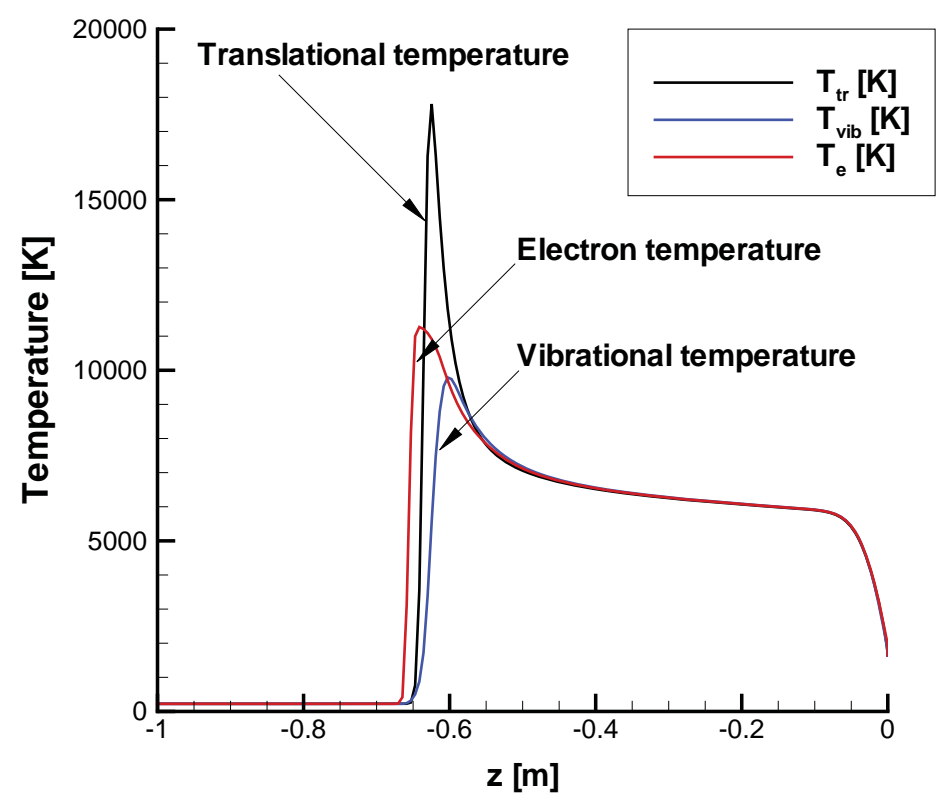

Figure 12. Temperature distribution along stagnation streamline of the ATV; $V_{\infty}=7329[\mathrm{~m} / \mathrm{s}] ; \rho_{N_{2}, \infty}=2.1667 \times$ $10^{-5}\left[\mathrm{~kg} / \mathrm{m}^{3}\right] ; \rho_{O_{2}, \infty}=6.6157 \times 10^{-6}\left[\mathrm{~kg} / \mathrm{m}^{3}\right] ; T_{\infty}=223.9[\mathrm{~K}] ; T_{\text {wall }}=1600[\mathrm{~K}]$

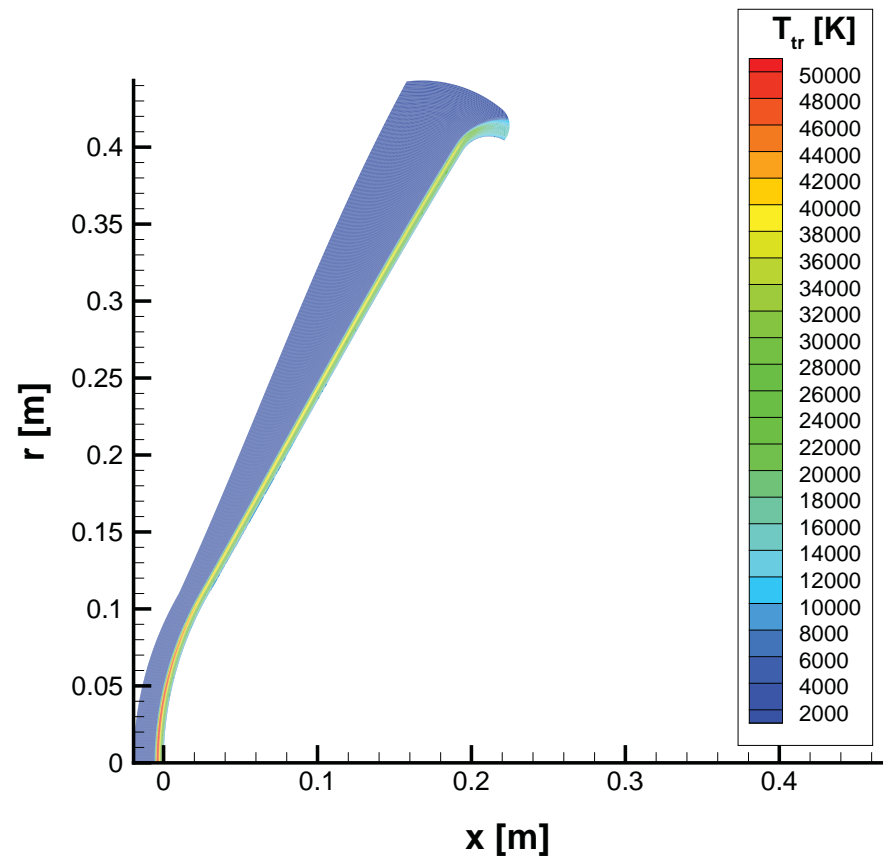

Figure 13. Contours of translational temperature around the Stardust at $64.7 \mathbf{~ k m}$ altitude; $V_{\infty}=11414[\mathrm{~m} / \mathrm{s}] ; \rho_{\infty}=$ $1.4 \times 10^{-4}\left[\mathrm{~kg} / \mathrm{m}^{3}\right] ; T_{\infty}=230.8[\mathrm{~K}] ; T_{\text {wall }}=4000[\mathrm{~K}]$ 


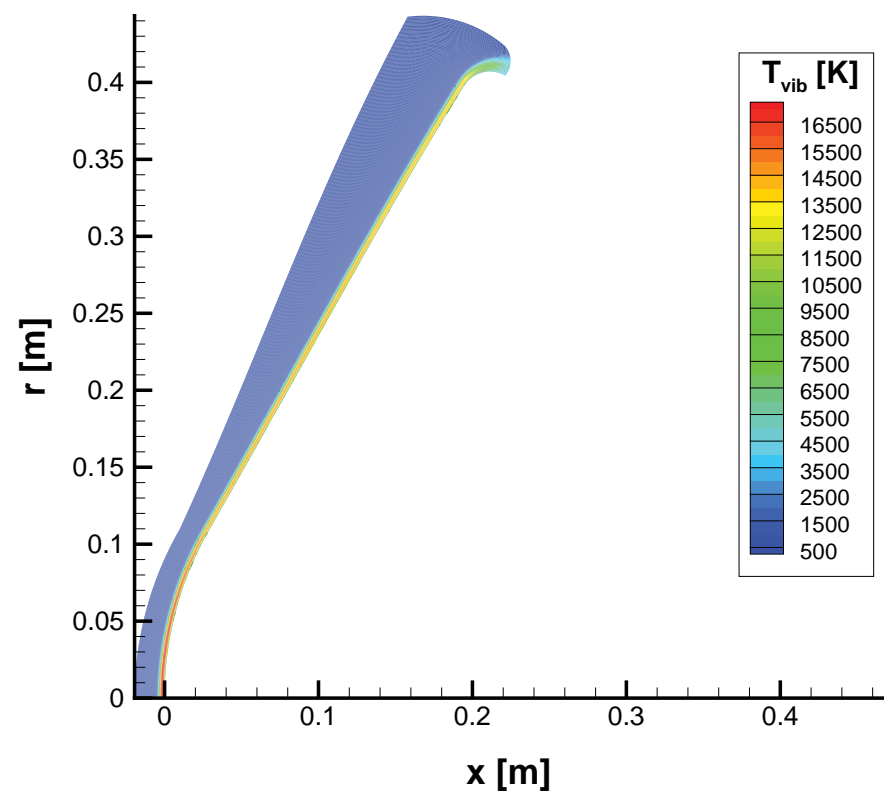

Figure 14. Contours of vibrational temperatures around the Stardust at $64.7 \mathrm{~km}$ altitude; $V_{\infty}=11414[\mathrm{~m} / \mathrm{s}] ; \rho_{\infty}=$ $1.4 \times 10^{-4}\left[\mathrm{~kg} / \mathrm{m}^{3}\right] ; T_{\infty}=230.8[\mathrm{~K}] ; T_{\text {wall }}=4000[\mathrm{~K}]$

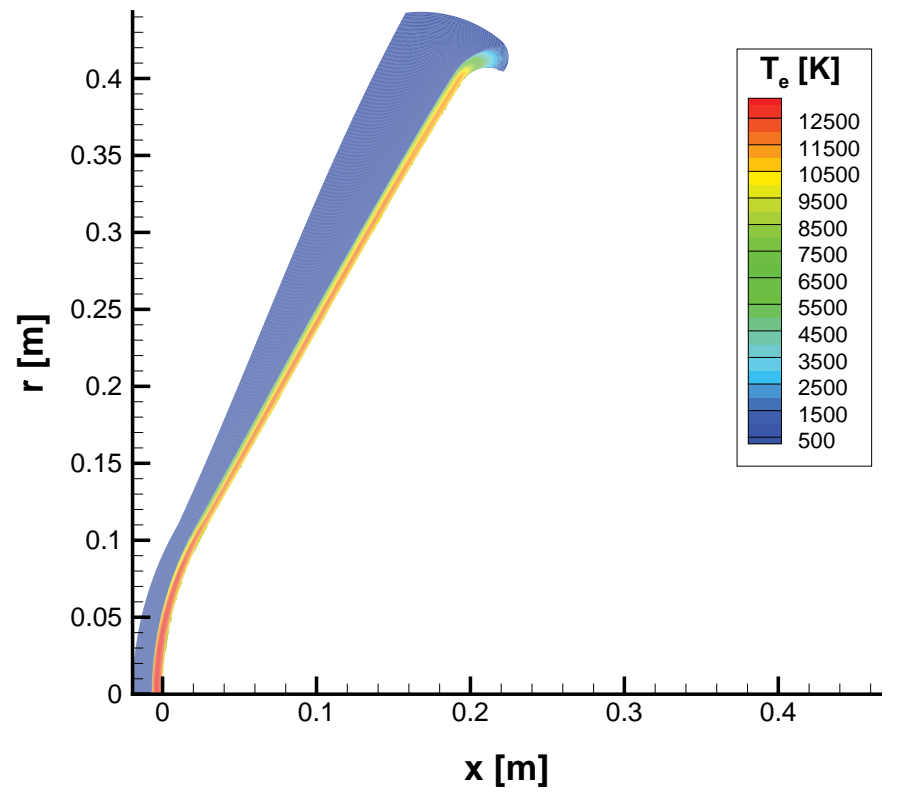

Figure 15. Contours of Electron temperatures around the Stardust at $64.7 \mathbf{~ k m}$ altitude; $V_{\infty}=11414[\mathrm{~m} / \mathrm{s}] ; \rho_{\infty}=$ $1.4 \times 10^{-4}\left[\mathrm{~kg} / \mathrm{m}^{3}\right] ; T_{\infty}=230.8[\mathrm{~K}] ; T_{\text {wall }}=4000[\mathrm{~K}]$ 


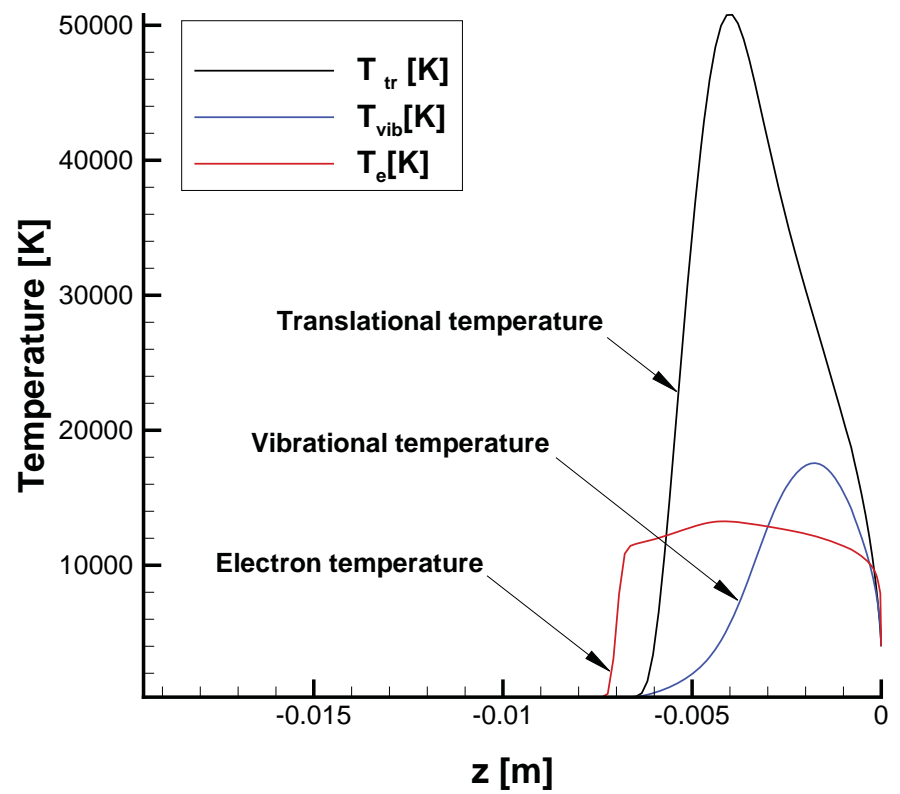

Figure 16. Temperature distributions along stagnation streamline of the Stardust at $\mathbf{6 4 . 7} \mathbf{~ k m}$ altitude; $V_{\infty}=11414[\mathrm{~m} / \mathrm{s}]$; $\rho_{\infty}=1.4 \times 10^{-4}\left[\mathrm{~kg} / \mathrm{m}^{3}\right] ; T_{\infty}=230.8[\mathrm{~K}] ; T_{\text {wall }}=4000[\mathrm{~K}]$ 


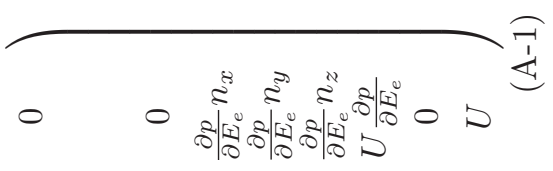

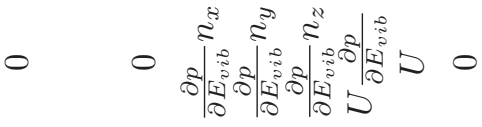

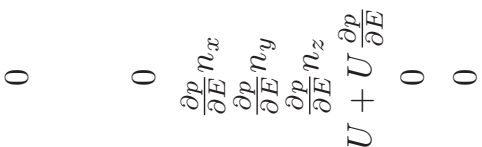

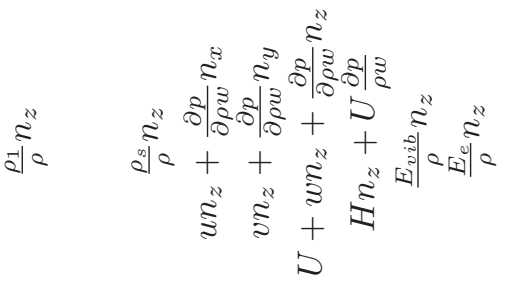

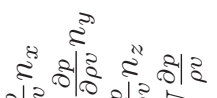

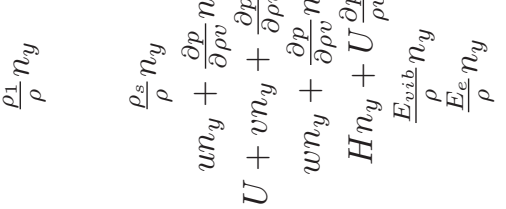

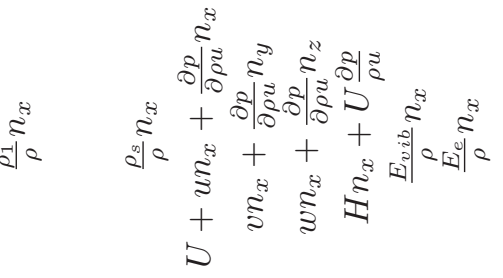

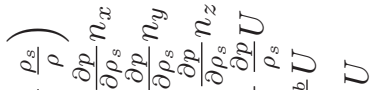

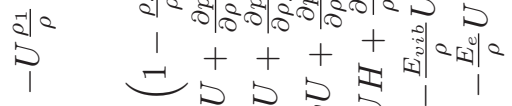

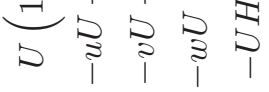

$\begin{array}{llllllll}\vdots & \vdots & \vdots & \vdots & \vdots & \vdots & \vdots & \vdots\end{array}$

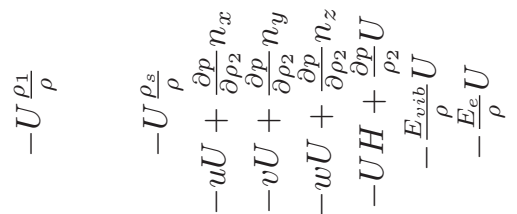

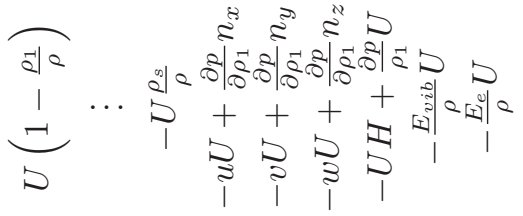

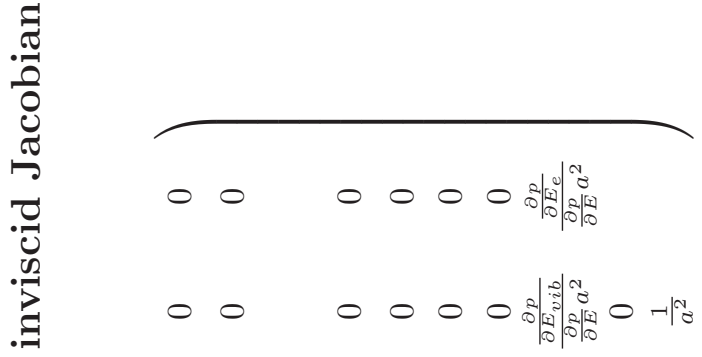

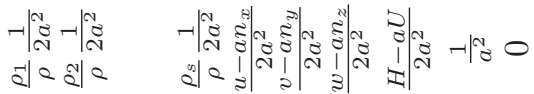

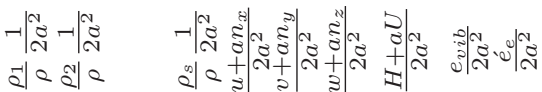

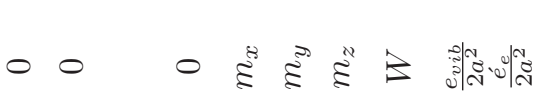

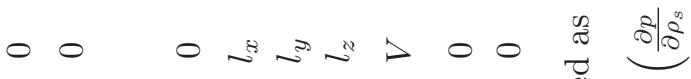

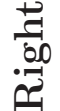

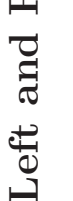

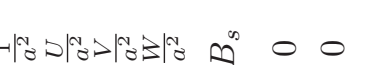

$\vdots i \vdots i$

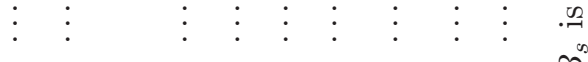

20

ñ

$0-\left.\right|^{2}: 3$

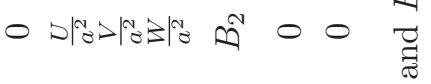

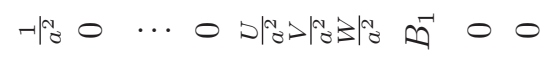

$\stackrel{4}{11}$

$\sqrt[8]{8}+\frac{8}{2}$
+

का

+ ขै

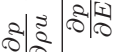

$\frac{1}{10 \mid 80}$

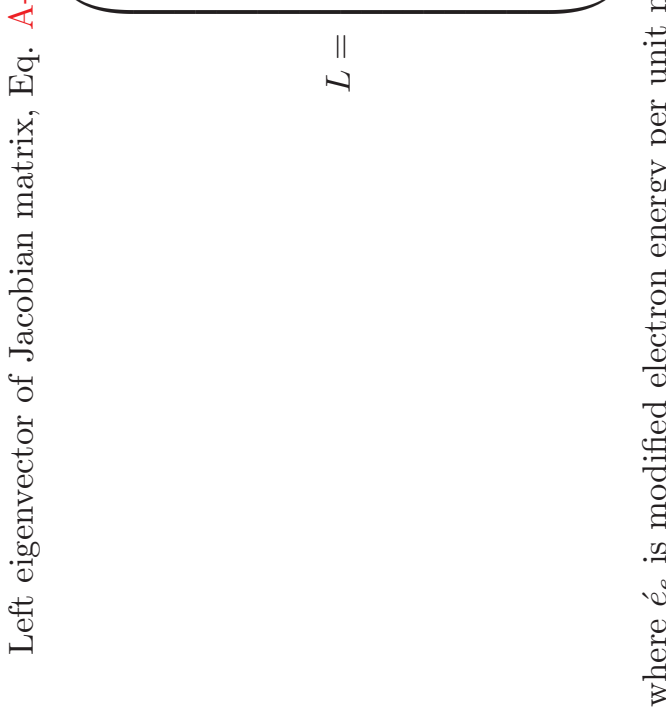

20 of 22 
重要

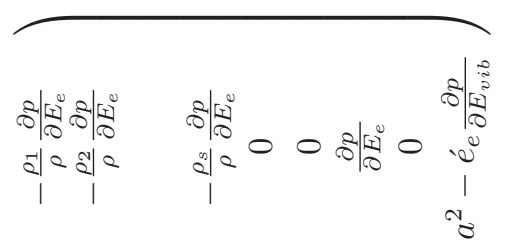

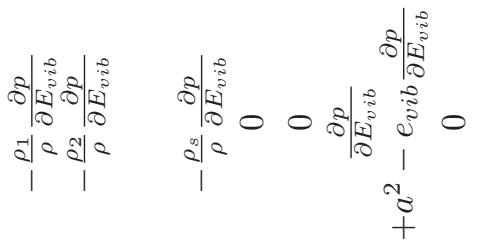

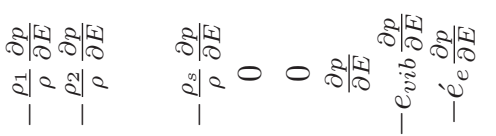

ช.

$0^{0}$
+

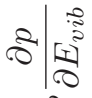

ปับ

$+$

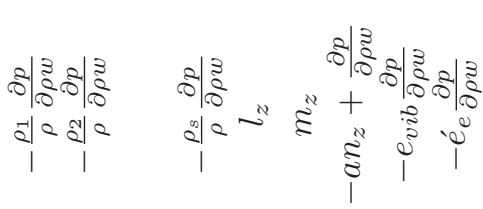

ริ|

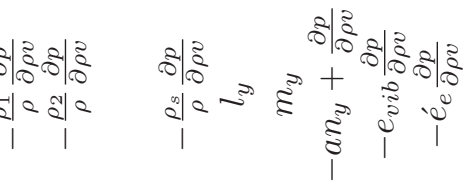

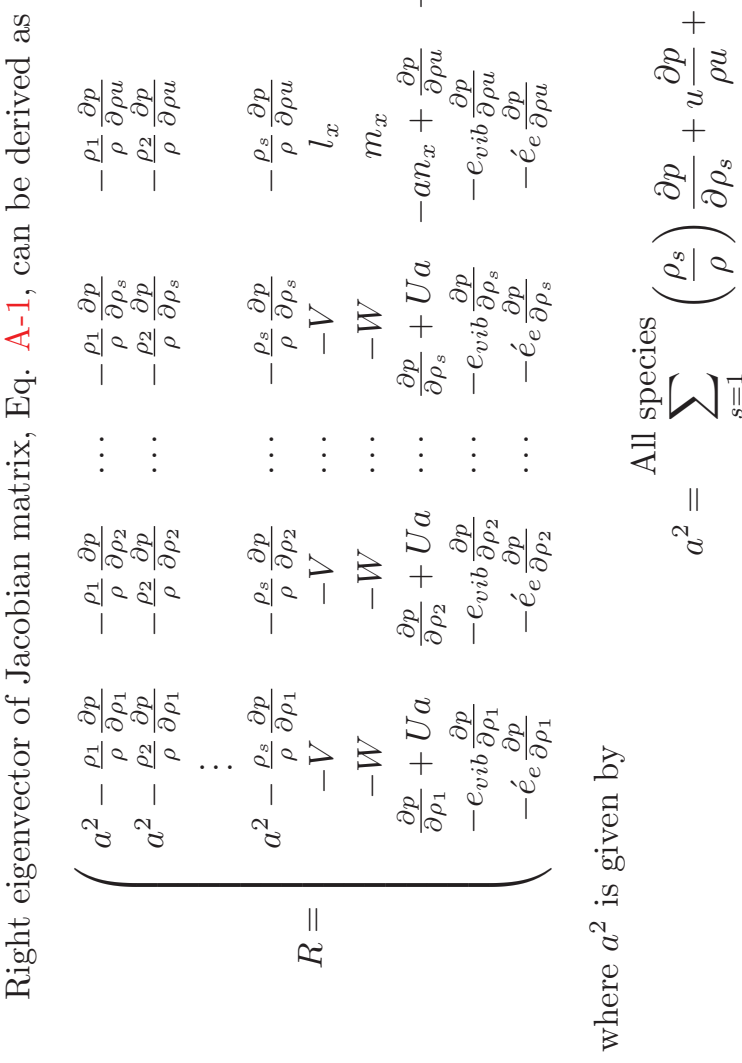

21 of 22 


\section{Acknowledgement}

M. Kim wishes to thank Alexandre Martin and Chul Park for useful discussion regarding numerical simulation and electron energy relaxation.

\section{References}

${ }^{1}$ Park, C., "On Convergence of Computation of Chemically Reacting Flows," AIAA 85-0247, January 1985, presented at the 23rd AIAA Science Meeting, Reno, Nevada.

${ }^{2}$ Lee, J. H., "Basic Boverning Equations for the Flight Regimes og Aeroassisted Orbital Transfer Vehicles," AIAA 84-1729, June 1984, presented at the 19th AIAA Thermoyphysics Conference, Snowmass, Colorado.

${ }^{3}$ Park, C., "Assesment of Two-Temperature Kinetics Model for Ionizing for Dissociating and Weakly Ionizing Nitrogen," Jouranl of Thermophysics Heat Transfer, Vol. 2, No. 1, 1989, pp. 8-16.

${ }^{4}$ Kim, M., Boyd, I. D., and Keidar, M., "Modeling of Electromagnetic Manipulation of Plasma for Communication during Re-entry Flight," Journal of Spacecraft and Rockets, Vol. 47, No. 1, 2010, pp. 29-35.

${ }^{5}$ Park, C., Nonequilibrium Hypersonic Aerothermodynamics, Wiley-Interscience, 1990.

${ }^{6}$ Candler, G. V. and MacCormack, R. W., "Computation of Weakly Ionized Hypersonic Flows in Thermochemical Nonequilibrium," Journal of Thermophysics and Heat Transger, Vol. 5, No. 3, 1991, pp. 266-273.

${ }^{7}$ Scalabrin, L. C. and Boyd, I. D., "Numerical Simulation of Weakly Ionized Hypersonic Flow for Reentry Configurations," AIAA 2006-3773, June 2006, presented at the 9th AIAA/ASME Joint Thermophysics and Heat Transfer Conference, San Francisco, California.

${ }^{8}$ Gnoffo, P. A., Gupta, R. N., and Shinn, J. L., "Conservation Equations and Physical Models for Hypersonic Air Flows in Thermal and Chemical Nonequilibrium," NASA STI/Recon Technical Report N, Vol. 89, Feb. 1989, pp. 16115-+.

${ }^{9}$ Candler, G. V. and MacCormack, R. W., "Computation of Weakly Ionized Hypersonic Flows in Thermochemical Nonequilibrium," Journal of Thermophysics and Heat Transfer, Vol. 5, No. 3, 1991, pp. 266-273.

${ }^{10}$ Josyula, E. and Bailey, W. F., "Governing Equations for Weakly Ionized Plasma Flowfields of Aerospace Vehicles," Journal of Spacecraft and Rockets, Vol. 40, No. 6, 2003, pp. 845-857.

${ }^{11}$ Lieberman, M. A. and Lichtenberg, A. J., Principles of Plasma Discharges and Materials Processing, Wiley-interscience, 2nd ed., 2005.

${ }^{12}$ Abe, H., Kihara, H., Uchida, T., and Nishida, M., "Experimental and Numerical Studies of Rotational Relaxation behind a Strong Shock Wave in Air," Shock Waves, Vol. 11, No. 6, 2002, pp. 413-421.

${ }^{13}$ Lazdinis, S. S. and Petrie, S. L., "Free electron and vibrational temperature nonequilibrium in high temperature nitrogen," Physics of Fluids, Vol. 17, Aug. 1974, pp. 1539-1546.

${ }^{14}$ Massey, H., Burhop, E., and Gilbody, E., Electronic and ionic impact phenomena, Oxford, 1967.

${ }^{15}$ Takayanagi, K., "Rotational and Vibrational Excitation of Polar Molecules by Slow Electrons," Journal of the Physical Society of Japan, Vol. 21, March 1966, pp. 507-+.

${ }^{16}$ Lee, J.-H., "Electron-Impact Vibrational Relaxation in High-Temperature Nitrogen," Journal of Thermophysics and Heat Transfer, Vol. 7, No. 3, 1993, pp. 399-405.

${ }^{17}$ Lee, J. H., "Electron-Impact Vibration Excitation Rates in the Flow Field of Aeroassisted Orbital Transfer Vehicles," AIAA 85-1035, June 1985, presented at the 20th AIAA Thermoyphysics Conference, Williamsburg, Virginia.

${ }^{18}$ Bourdon, A. and Vervisch, P., "Electron-vibration Energy Exchange Models in Nitrogen Plasma Flows," Phys. Rev. E, Vol. 55, No. 4, Apr 1997, pp. 4634-4641.

${ }^{19}$ Matsui, M., Komurasaki, K., and Arakawa, Y., "Characterization of Arcjet Type Arc-Heater Plumes," AIAA-2002-2242, May 2002, presented at the 33rd Plasmadynamics and Lasers Conference, Maui, Hawaii.

${ }^{20}$ Gülhan, A., Esser, B., Koch, U., Siebe, F., Riehmer, J., Giordano, D., and Konigorski, D., "Experimental Verification of Heat-Flux Mitigation by Electromagnetic Fields in Partially-Ionized-Argon Flows," Journal of Spacecraft and Rockets, Vol. 46, March 2009, pp. 274-283.

${ }^{21}$ Jones, W. L. and Cross, A. E., "Electrostatic Probe Measurements of Plasma Parameters for Two Re-entry Flight Experiments at 25000 feet-per-second," TIC D-6617, NASA, 1972.

${ }^{22}$ Anon., "Automated Transfer Vehicle Factshee," Tech. rep., European Space Agency, 2003, EUC-ESA-FSH-003.

${ }^{23}$ Trumble, K. A., Cozmuta, I., Sepka, S., and Jenniskens, P., "Post-flight Aerothermal Analysis of the Stardust Sample Return Capsule," 46th AIAA Aerospace Sciences Meeting and Exhibit, AIAA-2008-1201, Reno, NV, Jan. 7-10 2008. 